\title{
Potenciando el trabajo en equipo para un aprendizaje colaborativo en modalidad virtual
}

\section{Enhancing teamwork for collaborative learning in a virtual modality}

DOI: $10.46932 / s f j d v 3 n 1-021$

Received in: Dec 30st, 2021

Accepted in: Jan 1th, 2022

\section{Tania Gloria Tapia Opazo}

Químico de la Pontificia Universidad Católica de Chile, Magister en Ciencias de Recursos Naturales de la Universidad de La Frontera-Chile

Universidad de La Frontera-Chile

Av. Francisco Salazar 01145, Temuco-Chile

E-mail: tania.tapia@ufrontera.cl

Andrea de las Mercedes Arias Padilla

Ingeniero en Alimentos de la Universidad de La Frontera-Chile, Magíster en Didáctica de las Ciencias Experimentales de la Universidad Católica de Valparaíso-Chile

la Universidad de La Frontera-Chile

Av. Francisco Salazar 01145, Temuco-Chile

E-mail: andrea.arias@ufrontera.cl

Diego Eduardo Leiva Tapia

Estudiante de Pedagogía en Inglés, Universidad de La Frontera

Universidad de La Frontera

Av. Francisco Salazar 01145, Temuco-Chile

E-mail: d.leiva02@ufromail.cl

Sofía Cristina Leiva Tapia

Estudiante de Sociología, Universidad de La Frontera

Universidad de La Frontera

Av. Francisco Salazar 01145, Temuco-Chile

E-mail: s.leiva05@ufromail.cl

\section{RESUMEN}

El trabajo en equipo es una necesidad permanente, ya que el avance e innovación en las distintas áreas de desarrollo requiere una interdisciplinaridad que nos permita potenciar el conjunto de las áreas de trabajo. En este contexto, se aplicó una metodología organizacional de trabajo en equipo para estudiantes de primer y segundo año, en las asignaturas de Química General y Química Orgánica, de la Universidad de La Frontera, Temuco, Chile; con el objetivo de promover el trabajo y aprendizaje colaborativo en modalidad virtual. Se aplicó a un total de 230 estudiantes durante 3 semestres, en los años 2020 y 2021. La recolección de información se realizó mediante la aplicación de 2 encuestas de apreciación, una con respecto a la metodología aplicada y la otra con respecto al trabajo de pares dentro del equipo. Se pudo concluir que la metodología tuvo una buena recepción por parte de los estudiantes, la consideraron sencilla, adecuada y les permitió optimizar su tiempo de trabajo. Por otra parte, también manifestaron una buena apreciación del trabajo colaborativo interno de los equipos. 
palabra clave: Trabajo En Equipo, Aprendizaje Colaborativo, Química, Modalidad Virtual .

\begin{abstract}
Teamwork is a permanent need, since advancement and innovation in the different areas of development requires an interdisciplinary approach that allows us to enhance all work areas. In this way, an organizational teamwork methodology was applied for first and second year students, in the subjects of General Chemistry and Organic Chemistry, of the University of La Frontera, Temuco, Chile; with the aim of promoting collaborative work and learning in virtual mode. It was applied to a total of 230 students during 3 semesters in the years 2020 and 2021. The information collection was carried out through the application of 2 appreciation surveys, one regarding the applied methodology and the other regarding peer work within the work team. It was possible to conclude that the methodology was well received by the students, who considered it simple, adequate and that it allowed them to optimize their working time. On the other hand, there was also a good appreciation of the internal collaborative work of the teams.
\end{abstract}

Keyword: Teamwork, Collaborative Learning, Chemistry, Virtual Modality.

\title{
1 INTRODUCCIÓN
}

Dentro de las competencias profesionales, distintas investigaciones permiten afirmar que el trabajo en equipo es una competencia altamente demandada, por lo tanto, potenciar y desarrollar las habilidades y actitudes propias de esta competencia es un compromiso de todos los actores del proceso educativo (Lerís et al., 2017).

La necesidad de formar equipos con integrantes que aporten desde sus distintas características personales, ha traspasado las organizaciones, adentrándose en el ámbito de la formación universitaria (Florentín, 2015). De esta forma, a la generación de equipos aleatorios o formados por criterios académicos, se van agregando otras características que permiten construir equipos heterogéneos, que también consideran el género, capacidad de colaboración, interés, entre otros, para conseguir equipos homogéneos entre sí en cuanto a la búsqueda de resultados, pero heterogéneos internamente, en relación a sus características individuales (Lerís et al., 2017).

Hoy en día, el trabajo colaborativo es un elemento esencial en cualquiera de las actividades de enseñanza aprendizaje, por este motivo la gran mayoría de acciones o estrategias innovadoras en los procesos de enseñanza aprendizaje consideran esta forma de trabajo (Pérez-López, 2014). Una característica del trabajo colaborativo, es que se considera al individuo, no como una persona aislada, sino como parte del grupo, que comparte objetivos y responsabilidades. Además, en conjunto, llevando a cabo la interacción, se realizan procesos de intercambio y construcción del conocimiento (Aguilar et al., 2015).

Según Johnson (1999), el trabajo colaborativo es un sistema organizacional que induce la influencia recíproca entre los integrantes de un equipo. Se desarrolla a través de un proceso gradual en el que cada miembro y todos se sienten mutuamente comprometidos con el aprendizaje de los demás generando una interdependencia positiva que no implique competencia. El aprendizaje colaborativo se 
adquiere a través del empleo de métodos de trabajo grupal caracterizado por la interacción y el aporte de todos en la construcción del conocimiento-(Aguilar, et al., 2015).

De esta forma el trabajo en equipo y el trabajo y aprendizaje colaborativo, van estrechamente unidos en su desarrollo. Por lo tanto, un buen trabajo en equipo, nos permite desarrollar simultáneamente un aprendizaje colaborativo.

\section{DESARROLLO}

La metodología aplicada se focaliza en facilitar la organización y orientación de equipos para un mejor desempeño en el trabajo conjunto, colaborativo, para potenciar o desarrollar habilidades con sus pares en cuanto a comunicación, respeto, tolerancia, responsabilidad, ayuda y colaboración, y así, esta modalidad de trabajo pueda adaptarse y proyectarse a otras instancias de trabajo.

Esta metodología fue aplicada a un total de 150 estudiantes de segundo año, y 80 estudiantes de primer año, de la Universidad de La Frontera, en las asignaturas de Química General y Química Orgánica respectivamente, durante 3 semestres académicos en los años 2020 y 2021, con clases en modalidades asincrónicas y sincrónicas. Al término de cada semestre se aplicó una encuesta de apreciación para evaluar la metodología y una coevaluación para evaluar la participación de los integrantes del equipo.

El trabajo se desarrolló a lo largo del semestre en cuatro etapas, cada una evaluada con pauta y con ponderación diferentes, considerando el grado de dificultad y tiempo estimado en la preparación de éste. Después de cada etapa hubo un tiempo de retroalimentación para analizar las observaciones y poder incorporar mejoras en la etapa siguiente.

Etapa 1. Distribución aleatoria de grupos de trabajo (5-8 estudiantes), dependiendo del número total del curso. Cada grupo debió coordinar al menos una reunión de trabajo para conocerse, asignarse roles prioritarios dentro del equipo, y como producto entregaron un archivo con información básica (obligatoria) y complementaria, con respecto a los integrantes.

Etapa 2. Se les asignó un artículo de investigación (paper científico), relacionado con la asignatura y su carrera de estudio. Con este documento de referencia, buscaron información para actualizar, complementar, aplicar, argumentar y debatir el artículo de inicio. Debían considerar un mínimo de 10 referencias, debidamente citadas según normas APA.

Etapa 3. Con toda la información recopilada, cada equipo debió seleccionar textos e imágenes, que permitieran obtener un trabajo coherente, con una introducción, objetivos, desarrollo, conclusiones, referencias y reflexión, y una extensión definida que también les permitiera mostrar una capacidad de síntesis. 
Etapa 4. Finalmente, entregaron como producto de la etapa anterior un archivo (ppt, video, etc.), que diera cuenta del trabajo realizado, donde se evidenciara la presencia en voz o imagen de cada integrante del equipo.

\section{RESULTADOS}

Los resultados de esta metodología por una parte consideró la apreciación del profesor con respecto al desarrollo del trabajo durante el semestre, y por otra, la recolección de información en encuestas de apreciación de los estudiantes con respecto a la metodología de trabajo, además de una coevaluación para evaluar el trabajo de pares al interior del equipo.

Tabla $\mathrm{N}^{\circ} 1$. Encuesta de apreciación con respecto a la metodología organizacional

\begin{tabular}{|l|l|l|l|l|l|}
\hline Pregunta Nunca & $\begin{array}{l}\text { Casi } \\
\text { nunca }\end{array}$ & $\begin{array}{l}\text { A } \\
\text { veces }\end{array}$ & $\begin{array}{l}\text { Casi } \\
\text { siempre }\end{array}$ & Siempre \\
\hline $\begin{array}{l}\text { ¿Habías trabajado con esta forma de organización } \\
\text { grupal en otras instancias? (conocimiento de los } \\
\text { integrantes, asignación de roles, etc) }\end{array}$ & & & & \\
\hline $\begin{array}{l}\text { ¿Crees que fue adecuada la organización para } \\
\text { optimizar tiempo de dedicación, obtención de } \\
\text { resultados, entre otros? }\end{array}$ & & & & & \\
\hline $\begin{array}{l}\text { ¿La comunicación con tus pares y profesora fue } \\
\text { expedita y oportuna? }\end{array}$ & & & & & \\
\hline $\begin{array}{l}\text { ¿Crees que la retroalimentación y el tiempo asignado } \\
\text { para ello fueron útiles yertinentes? }\end{array}$ & & & & & \\
\hline $\begin{array}{l}\text { ¿Crees que fue más fácil trabajar con esta organización } \\
\text { que con otras utilizadas anteriormente? }\end{array}$ & & & & & \\
\hline $\begin{array}{l}\text { ¿Crees que sería conveniente y adecuado aplicar esta } \\
\text { organización de trabajo en futuros trabajos en equipo? }\end{array}$ & & & & & \\
\hline
\end{tabular}

Tabla N². Encuesta de coevaluación del trabajo al interior de cada equipo.

\begin{tabular}{|c|c|c|c|c|c|}
\hline Pregunta & Nunca & $\begin{array}{l}\text { Casi } \\
\text { nunca }\end{array}$ & A veces & $\begin{array}{l}\text { Casi } \\
\text { siempre }\end{array}$ & Siempre \\
\hline $\begin{array}{l}\text { ¿Su compañer@ participó en la confección de los } \\
\text { trabajos? }\end{array}$ & & & & & \\
\hline $\begin{array}{l}\text { ¿Su compañer@ presentó las excusas oportunamente } \\
\text { cuando no pudo cumplir con una asistencia o labor } \\
\text { asignada? }\end{array}$ & & & & & \\
\hline $\begin{array}{l}\text { ¿Su compañer@ respetó la organización y los } \\
\text { acuerdos del grupo? }\end{array}$ & & & & & \\
\hline $\begin{array}{l}\text { ¿Su compañer@ favoreció un buen ambiente de } \\
\text { trabajo? }\end{array}$ & & & & & \\
\hline $\begin{array}{l}\text { ¿Usted cree que su compañer@ fue un aporte, y } \\
\text { colaboró para un mejor trabajo en equipo? }\end{array}$ & & & & & \\
\hline
\end{tabular}

De acuerdo a la recopilación de información de las encuestas, reflejadas en el gráfico $\mathrm{N}^{\circ} 1$, podemos indicar que en cuanto a la organización de los grupos, sobre el $50 \%$ del total de estudiantes nunca la había desarrollado con la modalidad planteada, es decir, asignándose roles prioritarios 
consensuados y específicos de trabajo, dándole una identidad al equipo, con nombre y conocimiento de algunos elementos personales de cada integrante.

Sobre el $80 \%$ de los estudiantes manifestaron que el trabajo fue más fácil de realizar, ya que optimizaron sus tiempos de reuniones, que la comunicación con pares y profesora fue oportuna y expedita, que la retroalimentación fue eficiente y necesaria para mejorar cada etapa de trabajo, que además es una modalidad adecuada y conveniente de implementar en futuros trabajos en equipo.

Gráfico $\mathrm{N}^{\circ}$ 1. Porcentajes de respuestas favorables, con respecto a la metodología aplicada

\section{Apreciación de la metodología aplicada}

¿Habías trabajado con esta forma organización grupal en otras instancias? (conocimiento de los...

¿Crees que fue adecuada la organización para optimizar tiempo de dedicación, obtención de...

¿La comunicación con tus pares y profesora fue expedita y oportuna?

¿Crees que la retroalimentación y el tiempo asignado para ello fueron útiles y pertinentes?

¿Crees que fue más fácil trabajar con esta organización, que con otras utilizadas anteriormente?

¿Crees que sería conveniente y adecuado aplicar esta organización de trabajo en futuros trabajos en equipo?

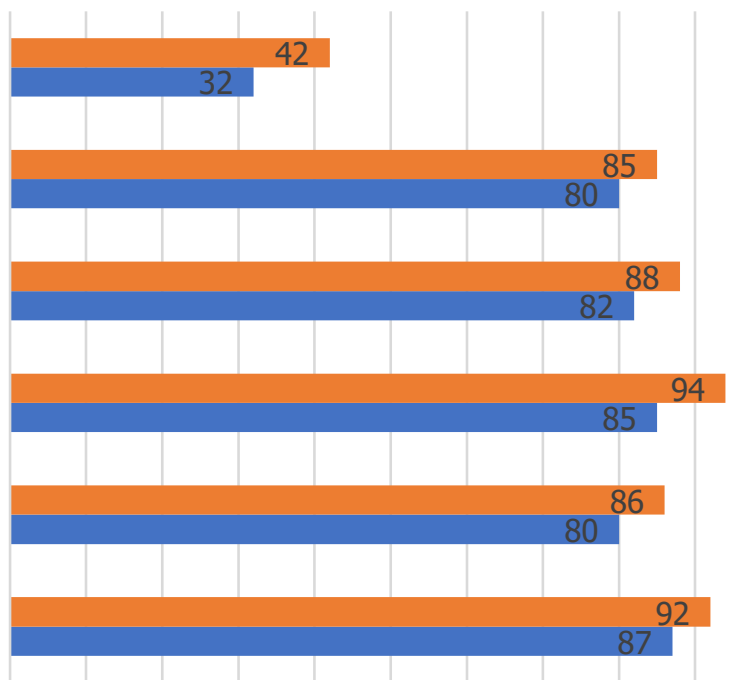

$\begin{array}{lllllllllll}0 & 10 & 20 & 30 & 40 & 50 & 60 & 70 & 80 & 90 & 100\end{array}$

- Química Orgánica @ Química General

Por otra parte, en cuanto al trabajo al interior del grupo, en el gráfico $\mathrm{N}^{\circ} 2$, el $45 \%$ del total de equipos de trabajo manifestó una total satisfacción del trabajo con sus pares, en la participación, en el aporte, responsabilidad, tolerancia, consenso, clima de trabajo y colaboración para un mejor trabajo en conjunto. 
Gráfico $\mathrm{N}^{\circ}$ 2. Porcentajes de respuestas frente al trabajo al interior del equipo.

\section{Coevaluación de pares}

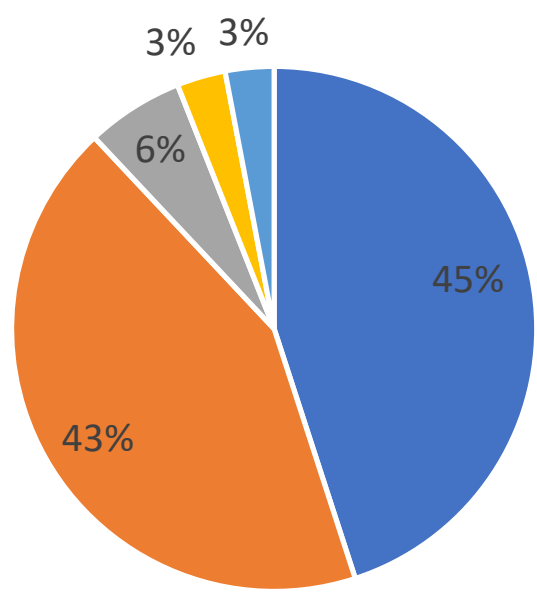

- Siempre $\square$ Casi siempre $\square$ A veces $\square$ Casi nunca $\square$ Nunca

No obstante, por diferentes motivos fue necesario realizar adecuaciones y reagrupaciones en algunos equipos de trabajo: abandono de la carrera, abandono de la asignatura, resistencia al trabajo en equipo, entre otros. En estas situaciones, se abordó en primer lugar una conversación con el equipo, para intentar resolver los conflictos presentados y cuando no fue posible hubo reagrupación de éstos.

Finalmente, se observa que los estudiantes de primer año, en la asignatura de Química Orgánica, tuvieron menor dificultad para abordar la metodología organizacional que los estudiantes de segundo año en la asignatura de Química General.

\section{CONCLUSIONES}

La metodología aplicada en la organización de equipos de trabajo, ha resultado efectiva y con una buena recepción por parte de los estudiantes, considerándola sencilla, adecuada y efectiva para optimizar el tiempo de dedicación a cada trabajo, generando un espacio propicio para intercambiar ideas, consensuar y colaborar con el aprendizaje entre pares.

Esta modalidad fue aplicada en tiempo de pandemia, con actividades completamente remotas, sincrónicas y asincrónicas, y en este escenario fue pertinente para la elaboración y desarrollo de los trabajos, la comunicación fluida y expedita entre estudiantes y entre estudiantes y profesora.

También es posible indicar que en los estudiantes de primer año, se aprecia una menor dificultad para abordar la metodología organizacional, y esto se puede explicar, ya que todos están en condiciones equivalentes de avance en su carrera, comparten los mismos horarios y su asignatura es exclusiva para la carrera, a diferencia de aquellos de segundo año, donde 5 carreras comparten la asignatura, lo que conlleva 
diferencias horarios, de carga académica, intereses propios de su carrera, además de tener al menos un año previo de permanencia en la universidad.

Por último indicar, que como proyección de esta metodología de trabajo en equipo es necesario realizar un seguimiento en cursos superiores.

\section{AGRADECIMIENTOS}

Departamento de Ciencias Químicas y Recursos Naturales, Universidad de La Frontera Dirección de carrera de Sociología, Universidad de La Frontera 


\section{REFERENCIAS}

Aguilar, N., Cedillo, M., Valenzuela, J. (2015) Logro de aprendizajes significativos a través de la competencia transversal "trabajo colaborativo" en educación superior. Voces y Silencios: Revista Latinoamericana de Educación, 6(1), 22-32.

Florentín, P. (2015). La competencia de Trabajo en Equipo en la Facultad de Educación de la Universidad de Zaragoza: análisis y estrategias para su mejora. (Tesis doctoral inédita). Zaragoza: Universidad de Zaragoza.

Johnson, D. y Johnson, R. (1999). Aprender juntos y solos. Aprendizaje cooperativo competitivo e individualista. Buenos Aires: Grupo Editorial Aique. http://terras.edu.ar/jornadas/3/biblio/3JOHNSONDavid-JOHNSON-Roger-Apendice.pdf

Lerís, D., Letosa, J., Usón, A., Allueva, P. y Bueno, C. (2017). Trabajo en equipo y estilos de aprendizaje en la educación superior. Revista Complutense de Educación, 28 (4), 1267-1284.

Pérez - López, Esteban (2014). Implementación de la estrategia didáctica del aprendizaje colaborativo, en el curso de laboratorio de técnicas instrumentales de análisis de la carrera de laboratorista químico. InterSedes: Revista de las Sedes Regionales, XV(32), 85-96. [fecha de Consulta 6 de Agosto de 2021]. ISSN: 2215-2458. Disponible en: https://www.redalyc.org/articulo.oa?id=66633023006 